\title{
Pancreatic Body Fracture does not Necessarily Imply Loss of Ductal Integrity Following Blunt Trauma Abdomen -A Rare Image
}

\author{
Disha Syal $^{1}$, Arindam Ghosh ${ }^{2}$ \\ ${ }^{1}$ Department of Surgery, Punjab Institute of Medical Sciences, Jalandhar, India \\ ${ }^{2}$ Department of Surgery, SPS Apollo Hospitals, Ludhiana, India \\ E-mail: dishasood@yahoo.com \\ Received February 28, 2011; revised April 27, 2011; accepted May 10, 2011
}

\begin{abstract}
Pancreatic injuries can result in significant morbidity and mortality if missed or unnoticed and also pose a great challenge in terms of diagnosis and treatment. We present a case of an 11 year old boy presenting with history of blunt trauma abdomen following handle bar injury. He had signs of abdominal distension and CECT of the abdomen showed transection of the pancreas in the region of junction between the body and tail. On exploratory laparotomy there was rupture at junction of body and tail of pancreas with intact pancreatic duct and a spleen preserving distal pancreatectomy was performed. In the presence of an intact pancreatic duct conservative management should be offered however this patient underwent spleen preserving distal pancreatectomy because of pancreatitis and necrosis ensuing in the pancreatic remnant margin. Hence, complete transection does not necessarily mean loss of ductal integrity and decision to preserve or remove the distal pancreas should not be based merely on ductal integrity but probably other factors also i.e. pancreatitis and necrosis.
\end{abstract}

Keywords: Blunt Trauma Abdomen, Pancreatic Body Fracture, Management, Intact Pancreatic Duct

\section{Case Report}

An 11 year old boy presented with history of blunt trauma abdomen following handle bar injury while riding a bicycle and developed pain in right upper abdomen, vomiting followed by abdominal distension. On examination he had pulse rate of 102 /minute with tenderness and guarding in epigastrium and left hypochondrium and generalized abdominal distension.

He was investigated with complete blood counts, liver function tests and renal function tests which were normal. A contrast enhanced Computerized tomography (CECT) of the abdomen was performed which showed transection of the pancreas in the region of junction between the body and tail. Moderate amount of free fluid was present mainly in the pelvis (Figure 1).

He underwent exploratory laparotomy which revealed peritonitis with saponification, mild hemoperitoneum, hematoma in transverse mesocolon, rupture at junction of body and tail of pancreas with intact pancreatic duct (Figure 2), splenic artery and vein. A spleen preserving distal pancreatectomy was performed (Figure 3). Following this patient had an uneventful recovery.

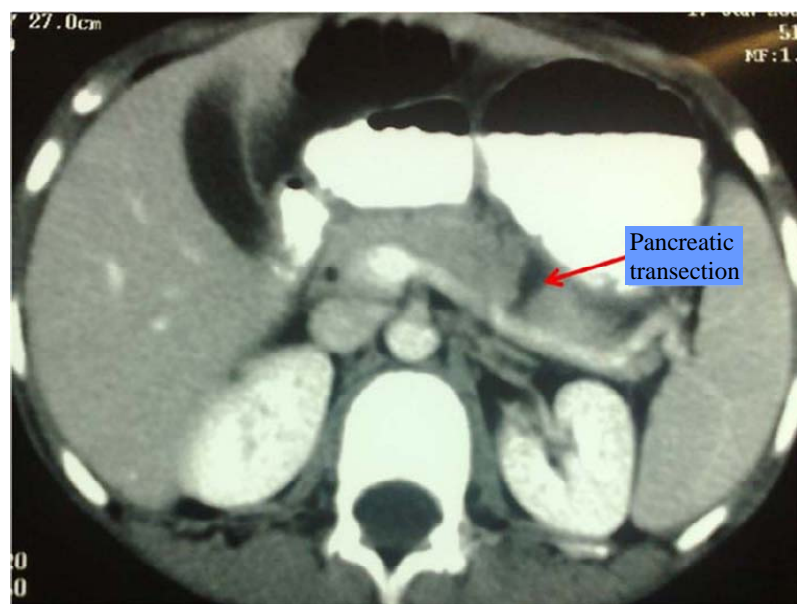

Figure 1. Contrast enhanced CT scan of the abdomen showing complete transection at the junction of body and tail of pancreas. 


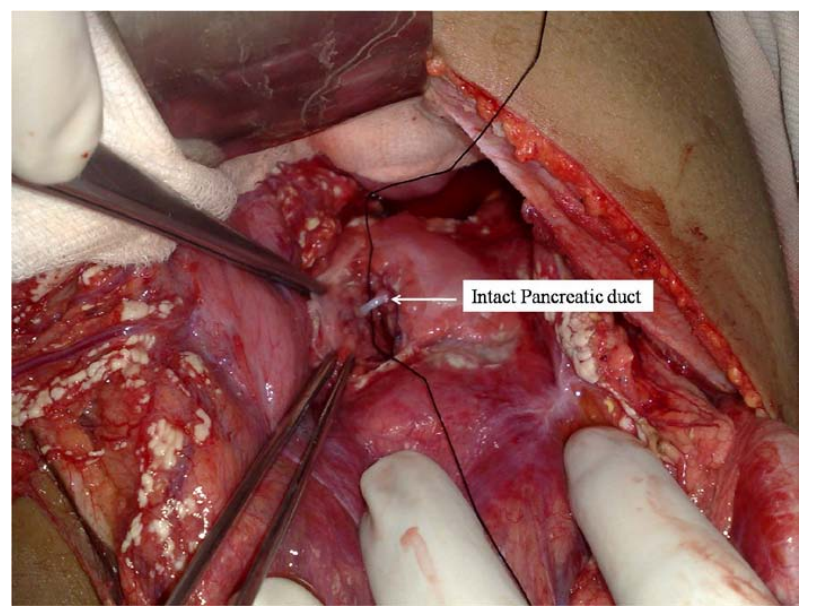

Figure 2. Complete transection of pancreas at junction of body and tail with an intact pancreatic duct.

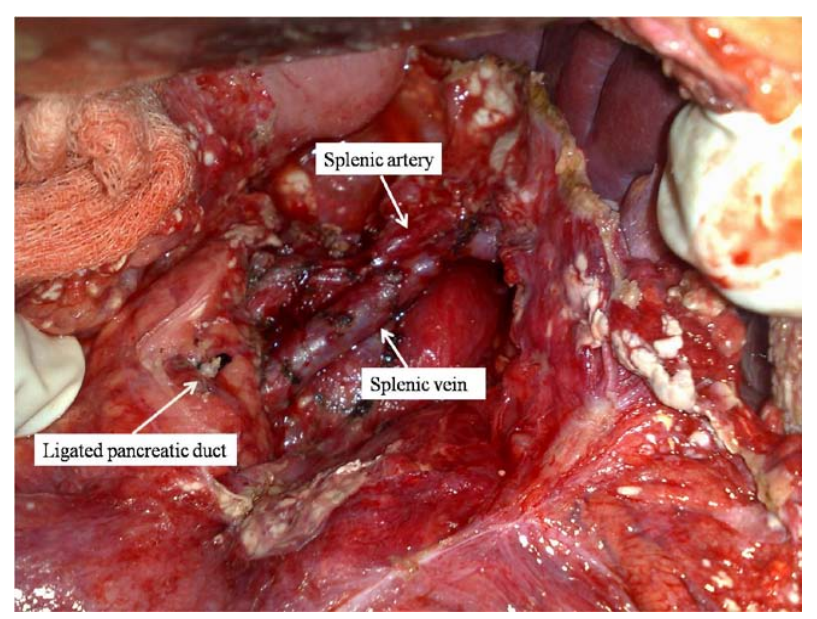

Figure 3. Spleen preserving distal pancreatectomy with ligated pancreatic duct.

\section{Discussion}

Following blunt trauma abdomen, the incidence of pancreatic injuries is very low as compared to other solid viscera injuries with rates of less than $1 \%$ reported of all trauma admissions [1]. The overall mortality rate reported for blunt pancreatic trauma varies between $16 \%$ to $20 \%$ [2]. Optimal management whether operative or nonoperative depends on the grade of pancreatic injury and ductal integrity.

This patient underwent exploration in view of peritoneal signs and although the pancreatic duct was intact, spleen preserving distal pancreatectomy was performed. Traditionally, in the presence of an intact pancreatic duct conservative management should be offered, this patient underwent spleen preserving distal pancreatectomy because of pancreatitis and necrosis ensuing in the pancreatic remnant margin. Hence, complete transection does not necessarily mean loss of ductal integrity and decision to preserve or remove the distal pancreas should not be based merely on ductal integrity but probably other factors also i.e. pancreatitis and necrosis.

\section{References}

[1] R. Akhrass, M. B. Yaffe, C. P. Brandt, M. Reigle, W. F. Fallon Jr. and M. A. Malangoni, "Pancreatic Trauma: A Ten-Year Multi-Institutional Experience,” The American Journal of Surgery, Vol. 63, No. 7, 1997, pp. 598-604.

[2] D. H. Wisner, R. L. Wold and C. F. Frey, "Diagnosis and Treatment of Pancreatic Injuries," Archives of Surgery, Vol. 125, No. 9, 1990, pp. 1109-1113. 